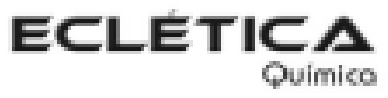

www.scielo.br/eq

Volume 29, número 1, 2004

\title{
Aplicação da zeólita natural escolecita na remoção de metais pesados de efluentes industriais: competição entre os cátions e processo de dessorção
}

\author{
S. M. Dal Bosco ${ }^{1}$, R. S. Jimenez ${ }^{1}$, W. A. Carvalho ${ }^{1}$ \\ ${ }^{I}$ Faculdade de Química - Centro de Ciências Exatas, Ambientais e de Tecnologias - Pontifícia Universidade Católica de \\ Campinas CEP 13086-900 - Campinas - SP - Brasil
}

\begin{abstract}
Resumo: Efluentes aquosos industriais são a principal causa de contaminação das águas com metais pesados. Diante de uma legislação cada vez mais rígida para o descarte desses metais, o desenvolvimento de procedimentos eficientes e de baixo custo para o tratamento de efluentes contendo metais pesados torna-se de grande interesse. Um estudo sobre a capacidade de retenção de metais pesados pela zeólita natural escolecita foi realizado, de modo a se avaliar a viabilidade desta aplicação. Os cátions utilizados foram $\mathrm{Mn}(\mathrm{II}), \mathrm{Cd}(\mathrm{II}), \mathrm{Ni}(\mathrm{II})$ e $\mathrm{Cr}(\mathrm{III})$. Nesta etapa do trabalho, foi avaliado o comportamento da zeólita na adsorção seletiva de cátions presentes nos pares $\mathrm{Cd} / \mathrm{Mn}, \mathrm{Cr} / \mathrm{Ni}, \mathrm{Cr} / \mathrm{Cd}$ e Ni/Mn, bem como a possibilidade de dessorção dos cátions metálicos adsorvidos em sua estrutura. A escolecita apresentou uma seletividade que pode ser relacionada, na maioria dos casos, à valência e ao raio iônico das espécies hidratadas. Os cátions $\mathrm{Cr}$ (III) e Ni(II) foram fortemente adsorvidos, não podendo ser substancialmente removidos por troca com cátions sódio ou cálcio. Dos cátions testados, apenas o $\mathrm{Cd}(\mathrm{II})$ apresentou comportamento de adsorção e de dessorção atípicos, demonstrando uma elevada labilidade no processo de troca iônica.
\end{abstract}

Palavras-chave: troca iônica; metais pesados; escolecita.

\section{Introdução}

Os efluentes industriais são as principais causas de contaminação das águas com metais potencialmente tóxicos. Como estes metais são biocumulativos, há uma crescente exigência por parte da sociedade e de órgãos públicos no sentido de diminuir esta contaminação a níveis toleráveis pelos organismos sujeitos ao contato com tais contaminantes e, consequentemente, uma tendência em se aprovar uma legislação ambiental cada vez mais rigorosa. Um estudo para remoção de $\mathrm{Mn}(\mathrm{II}), \mathrm{Cd}(\mathrm{II}), \mathrm{Ni}(\mathrm{II})$ e $\mathrm{Cr}$ (III) foi realizado, utilizando-se a zeólita natural escolecita, que foi coletada no município de Morro Reuter, Estado do Rio Grande do Sul, Brasil, e que possui a seguinte fórmula química [4]: $\mathrm{Na}_{0,3} \mathrm{Ca}_{1,0} \mathrm{Al}_{2,0} \mathrm{Si}_{3,0} \mathrm{O}_{10} \cdot 3 \mathrm{H}_{2} \mathrm{O}$. A escolecita é uma zeólita microporosa, cujas dimensões dos canais são de 2,6 x 3,9 А [3], e que ocorre na Província Ígnea Continental do Paraná, associada às porções superiores dos derrames basálticos. As zeólitas ocorrem na natureza em quantidade suficiente para serem consideradas como um recurso mineral viável. Dos nove tipos de zeólitas encontradas no sul do Brasil (Bacia do Paraná), a escolecita é a segunda em abundância [11].

Zeólitas são minerais formados por uma estrutura tridimensional de tetraedros de $\mathrm{SiO}_{4}$ e $\mathrm{AlO}_{4}$. Possuem cavidades e canais regulares e de tamanho molecular, nos quais pode haver movimentação de moléculas de água. O desbalanceamento de cargas provocado pelo $\mathrm{Al}$ estrutural, que é trivalente, é compensado por cátions de metais alcalinos e alcalinos terrosos. Esta condição confere às zeólitas a propriedade de troca iônica [9]. Além disso, as zeólitas possuem uma elevada superfície específica interna devido a 
porosidade da rede, característica indispensável para uma alta capacidade de troca iônica. O processo de troca iônica ocorre entre duas ou mais fases. A transferência de íons de uma fase para outra depende da eletroneutralidade e é regulada pela concentração dos íons em ambas as fases e pela seletividade que a zeólita apresenta sobre os íons [5].

Para zeólitas com alta carga estrutural, ou seja, com alta concentração de alumínio (razão Si/ Al baixa), a seqüência de seletividade de troca iônica geralmente está associada ao raio iônico hidratado, tendo a zeólita preferência por cátions pequenos [6].

A remoção de metais de efluentes aquosos tem sido feita, na maior parte, pela precipitação na forma de hidróxidos por meio de adição de $\mathrm{CaO}$. Embora esse método seja relativamente simples e barato, gera um grande volume de rejeitos cuja disposição é perigosa e de alto custo, além de não produzir um efluente com conteúdo de metais pesados suficientemente baixo [12]. A aplicação de zeólitas naturais em escala industrial tem sido limitada à remoção de amônia de efluentes aquosos municipais e, em menor extensão, à remoção de $\mathrm{Cs}$ e $\mathrm{Sr}$ de efluentes radioativos [14]. Entretanto, devido a sua alta seletividade de troca, boa resistência física e química e excelente compatibilidade com o meio ambiente, as zeólitas constituem uma classe de adsorventes de grande interesse econômico e social, principalmente no que se refere a remoção de cátions de metais potencialmente tóxicos de efluentes industriais.

\section{Materiais e métodos}

Para a quantificação dos metais, com exceção do Ni(II), utilizou-se a técnica de espectrofotometria de absorção atômica, em um espectrofotômetro Perkin Elmer Analyst 300. Para a construção das curvas analíticas, foram obtidas amostras de soluções dos metais com diferentes concentrações, a partir de uma solução padrão de $1000 \mathrm{mg} \mathrm{L}^{-1}$ de cada metal (Titrisol ${ }^{\circledR}$, Merck). As concentrações das amostras variaram de $0,5 \mathrm{mg} \mathrm{L}^{-1}$ a $5,0 \mathrm{mg} \mathrm{L}^{-1}$.

O Ni(II) foi quantificado por método colorimétrico [18], que utiliza alíquota das soluções a serem analisadas, $1 \mathrm{~mL}$ de água de bromo saturada, $2 \mathrm{~mL}$ de hidróxido de amônio concentrado, $1 \mathrm{~mL}$ de dimetilglioxima ( $1 \%$ em etanol) em um balão volumétrico de $10 \mathrm{~mL}$. A leitura foi feita após 5 minutos, no comprimento de onda de 445 $\mathrm{nm}$.

Para avaliar a competição entre os cátions no processo de adsorção, foram utilizados sistemas contendo dois íons diferentes. Foram testadas as duplas $\mathrm{Cd} / \mathrm{Mn}, \mathrm{Cr} / \mathrm{Ni}, \mathrm{Cr} / \mathrm{Cd}$ e Ni/Mn. Foram preparadas 5 soluções dos metais em concentrações variadas, perfazendo uma concentração total de $50 \mathrm{mg} \mathrm{L}^{-1}$ e um volume de $120 \mathrm{~mL}$. Os testes de adsorção foram conduzidos no $\mathrm{pH}$ original das soluções (3,5 para $\mathrm{Cr}$, 4,5 para $\mathrm{Cd}$ e 5,5 para $\mathrm{Ni}$ e $\mathrm{Mn})$. As soluções foram postas em contato com 2,0 g de escolecita e submetidas a agitação magnética constante, por 24 horas, a temperatura ambiente. Após os intervalos de tempo de 1, 2, 3, 4, 5, 12 e 24 horas, foram retiradas alíquotas de $5 \mathrm{~mL}$ do meio reacional com o auxílio de uma seringa de vidro. Para separar a fase líquida da sólida, foi acoplada à seringa um filtro RC15 com 0,2 mm de diâmetro de poro. As soluções foram diluídas para posterior quantificação.

Para o processo de dessorção, utilizou-se amostras de 1,0 g de escolecita previamente submetidas à troca iônica com $60 \mathrm{~mL}$ de soluções com concentração inicial de $50 \mathrm{mg}$. $\mathrm{L}^{-1}$ de cada metal. As amostras foram postas em contato com $100 \mathrm{~mL}$ de uma solução 2,0 $\mathrm{M}$ de $\mathrm{NaCl}$ ou de $\mathrm{Ca}\left(\mathrm{NO}_{3}\right)_{2}$. Alíquotas de $2,5 \mathrm{~mL}$ foram retiradas do meio reacional após 1, 2, 3, 4, 5 e 24 horas. Separou-se a fase sólida da líquida por meio de filtro RC 15 com $0,2 \mathrm{~mm}$, acoplado à seringa.

\section{Resultados e discussão}

As curvas analíticas dos quatro metais analisados apresentaram comportamento linear na faixa de concentração que vai até $5,0 \mathrm{mg} . \mathrm{L}^{-1}$, com os coeficientes de correlação mostrados na Tabela 1 . Portanto, esta faixa de concentração foi utilizada para quantificar as amostras.

Tabela 1 - Coeficientes de correlação das curvas de calibração.

\begin{tabular}{cc}
\hline Metal & Coeficiente de correlação \\
\hline $\mathrm{Mn}(\mathrm{II})$ & 0,9993 \\
$\mathrm{Cd}(\mathrm{II})$ & 0,9998 \\
$\mathrm{Ni}(\mathrm{II})$ & 0,9986 \\
$\mathrm{Cr}(\mathrm{III})$ & 0,9995 \\
\hline
\end{tabular}




\section{Competição entre os cátions}

A seletividade de zeólitas naturais pode ser avaliada em função do teor de alumínio em sua estrutura. Para zeólitas com baixa relação Si/Al, como a escolecita, temos uma carga estrutural relativamente elevada e, por conseqüência, uma preferência por cátions de maior valência e por cátions pequenos ou altamente carregados [5]. Este comportamento está relacionado à menor distância média entre sítios adjacentes aniônicos $\left(\mathrm{AlO}_{2}\right)$ na estrutura zeolítica, o que diminui a di- ficuldade de um cátion divalente em satisfazer os campos de dois sítios aniônicos adjacentes. A avaliação da energia de hidratação dos cátions também é importante, pois as dimensões dos poros da escolecita são inferiores aos diâmetros dos cátions hidratados, que estão relacionados na Tabela 2. Portanto, os cátions em solução aquosa devem perder parte de suas águas de hidratação para possibilitar sua entrada na estrutura da zeólita e, conseqüentemente, o acesso aos sítios de troca, o que acaba favorecendo os cátions com menor energia de hidratação.

Tabela 2 - Raio hidratado [13] e energia de hidratação [19] dos cátions testados.

\begin{tabular}{ccc}
\hline Cátion & Raio hidratado $(\AA)$ & Energia de hidratação $\left(\mathrm{kJ} \mathrm{mol}^{-1}\right)$ \\
\hline $\mathrm{Mn}(\mathrm{II})$ & 4,38 & 1845 \\
$\mathrm{Cd}(\mathrm{II})$ & 4,26 & 1806 \\
$\mathrm{Ni}(\mathrm{II})$ & 4,04 & 2106 \\
$\mathrm{Cr}(\mathrm{III})$ & 4,61 & 4402 \\
\hline
\end{tabular}

A preferência da zeólita por um dos cátions envolvidos na troca pode ser expressa pelo fator de separação $\alpha_{B}^{A}$, definido por:

$$
\alpha_{B}^{A}=\frac{X_{A(z)} \cdot X_{B(s)}}{X_{B(z)} \cdot X_{A(s)}}
$$

onde $\mathrm{X}_{(\mathrm{S})}$ e $\mathrm{X}_{(\mathrm{Z})}$ são as frações equivalentes dos cátions A ou B na solução e na zeólita, respectivamente.
Se o cátion A é preferido, > 1. Caso o cátion B seja preferido, isto implica $<1$. Logo, pode-se concluir que quanto maior o valor do fator de separação, maior será a preferência da zeólita pelo cátion A. Esta consideração é bastante importante na escolha de uma determinada zeólita para uma aplicação particular, pois quanto maior for o valor de , menor deverá ser a quantidade de zeólita a ser utilizada nos processos de remoção.

Os valores de a para todos os pares de cátions testados são apresentados na Tabela 3.

Tabela 3 - Valores de a para todos os pares de cátions testados.

\begin{tabular}{|c|c|c|c|c|}
\hline $\begin{array}{c}\text { Concentrações } \\
\text { iniciais }\left(\mathrm{mg} \mathrm{L}^{-1}\right)\end{array}$ & $\mathrm{Cd}(\mathrm{II}) / \mathrm{Mn}(\mathrm{II})$ & $\mathrm{Cr}(\mathrm{III}) / \mathrm{Cd}(\mathrm{II})$ & $\mathrm{Ni}(\mathrm{II}) / \mathrm{Mn}(\mathrm{II})$ & $\mathrm{Cr}(\mathrm{III}) / \mathrm{Ni}(\mathrm{II})$ \\
\hline $41,7 / 8,3$ & 9,1 & 1,2 & 9,3 & $2,2 \mathrm{E}+04$ \\
\hline $33,3 / 16,7$ & 29,0 & 0,44 & 2,4 & $5,9 \mathrm{E}+03$ \\
\hline $25,0 / 25,0$ & 10,4 & $1,0 \mathrm{E}-03$ & 1,2 & $2,1 \mathrm{E}+03$ \\
\hline $16,7 / 33,3$ & 2,6 & $2,9 \mathrm{E}-02$ & 1,0 & $4,2 \mathrm{E}+03$ \\
\hline $8,3 / 41,7$ & 7,4 & $5,9 \mathrm{E}-04$ & 0,91 & $4,2 \mathrm{E}+02$ \\
\hline
\end{tabular}


As cinéticas de adsorção para o par Cd(II)/ $\mathrm{Mn}$ (II) em duas concentrações iniciais diferentes (indicadas nas legendas) são apresentadas na Figura 1.

A Figura 1 e os valores de a superiores a 1,0 (Tabela 3) demonstram que o cátion $\mathrm{Cd}(\mathrm{II})$ é preferencialmente retido pela escolecita quando em

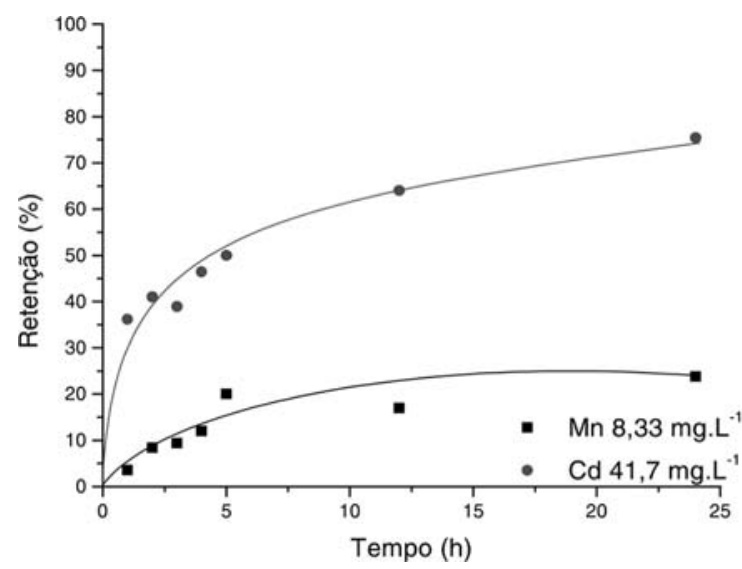

Figura 1 - Cinéticas de adsorção para o par Cd(II)/Mn(II).

As cinéticas de adsorção para o par $\mathrm{Cr}(\mathrm{III}) /$ $\mathrm{Ni}(\mathrm{II})$ em duas concentrações iniciais diferentes são apresentadas na Figura 2.

$\mathrm{O} \mathrm{Ni}(\mathrm{II})$ deveria ser adsorvido preferencialmente ao $\mathrm{Cr}(\mathrm{III})$, se considerarmos que seu raio hidratado e sua energia de hidratação são menores. Porém, deve-se considerar a maior força iônica
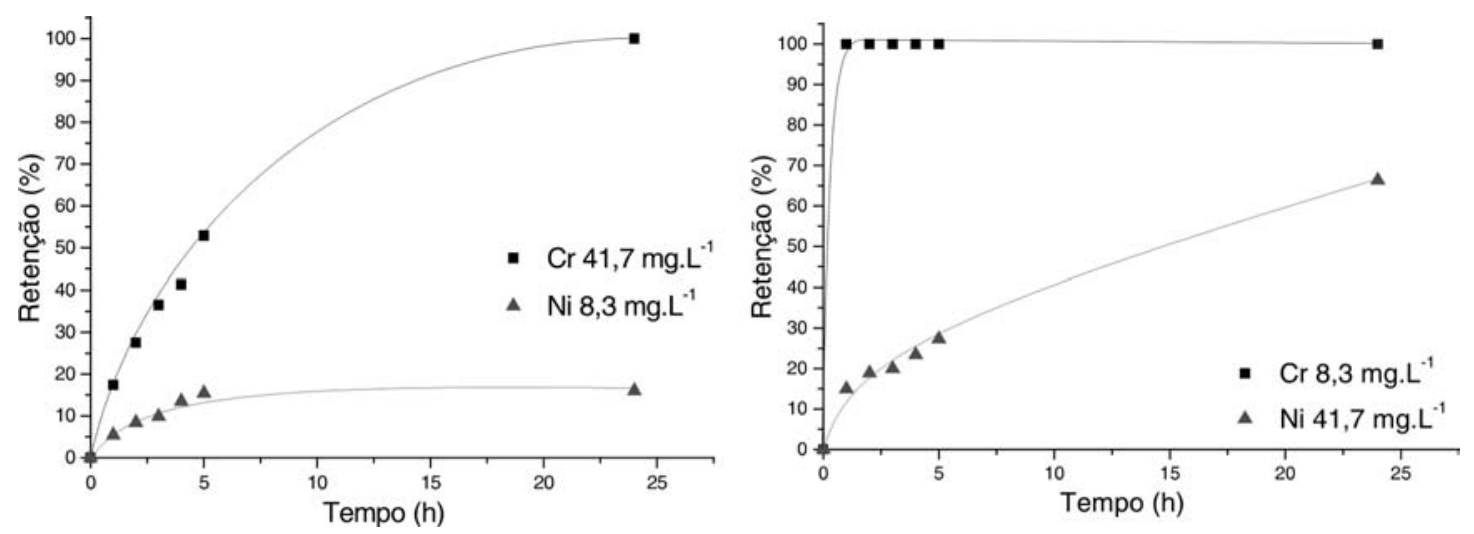

Figura 2 - Cinéticas de adsorção para o par $\mathrm{Cr}(\mathrm{III}) / \mathrm{Ni}(\mathrm{II})$. competição com o cátion $\mathrm{Mn}(\mathrm{II})$, para todos os valores de concentração testados. Embora a diferença entre os valores de raio hidratado e energia de hidratação entre os dois cátions seja pequena, o $\mathrm{Cd}(\mathrm{II})$ é favorecido em ambos os casos pois, além de possuir menor raio hidratado, necessita de menos energia para perder suas águas de hidratação.

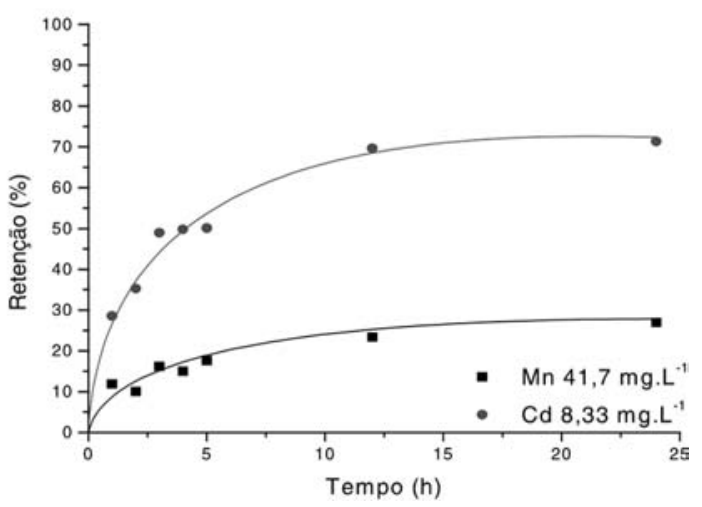

do cromo comparado com os outros cátions aqui testados, já que o cromo é o único cátion trivalente. Este favorecimento é bastante expressivo, uma vez que os valores de a para todas as concentrações testadas são muito superiores a 1,0, demonstrando que a escolecita tem uma preferência acentuada pelo $\mathrm{Cr}(\mathrm{III})$. 
As cinéticas mostram que, além de ser mais rápida, a adsorção do $\mathrm{Cr}$ (III) é total em todos os valores de concentração testados. No sistema com concentração inicial de $\mathrm{Cr}(\mathrm{III})$ de $41,7 \mathrm{mg} \mathrm{L}^{-1}$, a adsorção total só ocorre após $24 \mathrm{~h}$ de contato, enquanto um sistema com $25 \mathrm{mg} \mathrm{L}^{-1} \mathrm{de} \mathrm{Cr}$ (III) atinge o equilíbrio em apenas 3 horas. Com o Ni(II), a escolecita apresenta uma velocidade de troca inferior e se estabiliza com valores de adsorção bastante reduzidos.

As cinéticas de competitividade para o par $\mathrm{Ni}(\mathrm{II}) / \mathrm{Mn}$ (II) em duas concentrações iniciais diferentes são apresentadas na Figura 3. Podemos observar que o $\mathrm{Ni}$ (II) foi retido preferencialmente em quase todas as concentrações testadas, sendo o $\mathrm{Mn}$ (II) preferido apenas quando em concentração muito superior à do $\mathrm{Ni}$ (II) $\left(41,7 \mathrm{mg} \mathrm{L}^{-1}\right.$ e $8,3 \mathrm{mg} \mathrm{L}^{-}$ ${ }^{1}$, respectivamente). A diferença de retenção, de

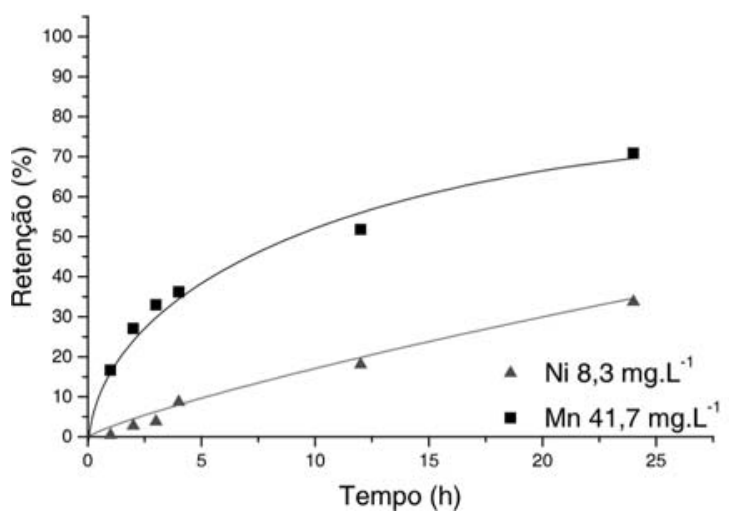

Figura 3 - Cinéticas de adsorção para o par Ni(II)/Mn(II).

As cinéticas de competitividade para o par $\mathrm{Cr}(\mathrm{III}) / \mathrm{Cd}(\mathrm{II})$ em duas concentrações iniciais diferentes são apresentadas na Figura 4. A escolecita tem preferência pelo $\mathrm{Cd}$ (II) quando a sua concentração inicial é superior ou igual à do $\mathrm{Cr}$ (III). De todas as concentrações testadas, a única na qual o $\mathrm{Cr}$ (III) foi preferido pela escolecita durante todo o período analisado foi quando a sua concentração inicial era 5 vezes maior do que a do $\mathrm{Cd}$ (II) e, mesmo assim, o fator de separação a é apenas ligeira- modo geral, não foi muito acentuada, como pode ser observado pelos valores de a próximos de 1 . Um ligeiro aumento no fator de separação só ocorre quando a concentração do $\mathrm{Ni}$ (II) é bastante superior à do $\mathrm{Mn}$ (II), ou seja, $41,7 \mathrm{mg} \mathrm{L}^{-1}$ e $8,3 \mathrm{mg} \mathrm{L}^{-1}$, respectivamente. Isto se deve ao fato dos valores de raio hidratado e de energia de hidratação dos cátions possuírem variações pequenas, quando comparados. Na competição, o Ni(II) é favorecido em relação ao $\mathrm{Mn}$ (II) pelo valor de raio hidratado, $4,04 \AA$ e $4,38 \AA$, respectivamente. Por outro lado, comparando-se os cátions quanto às suas energias de hidratação, o Mn(II) possui o menor valor para este parâmetro $\left(1845 \mathrm{~kJ} \mathrm{~mol}^{-1}\right.$ contra $2106 \mathrm{~kJ} \mathrm{~mol}^{-}$ ${ }^{1}$ do Ni(II)), sendo, portanto, preferido. Dessa forma, os dados experimentais demonstram que $o$ valor do raio hidratado foi o parâmetro determinante de seletividade neste caso.

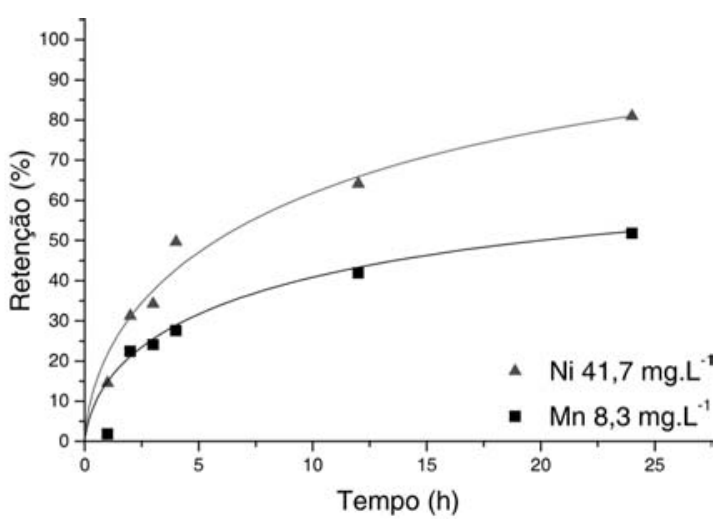

mente superior a 1 (Tabela 3). Neste caso, a preferência da escolecita não foi determinada pela maior valência do cátion, como seria esperado. Assim como o $\mathrm{Ni}(\mathrm{II})$, o $\mathrm{Cd}(\mathrm{II})$ possui tanto o valor de raio hidratado quanto de energia de hidratação menores do que os do $\mathrm{Cr}(\mathrm{III})$. Ressalta-se apenas que a diferença entre as energias de hidratação do $\mathrm{Cr}$ (III) e do Cd(II) é bastante expressiva (4402 $\mathrm{kJ} \mathrm{mol}^{-1} \mathrm{e}$ $1806 \mathrm{~kJ} \mathrm{~mol}^{-1}$, respectivamente) o que pode, em parte, explicar este comportamento. 

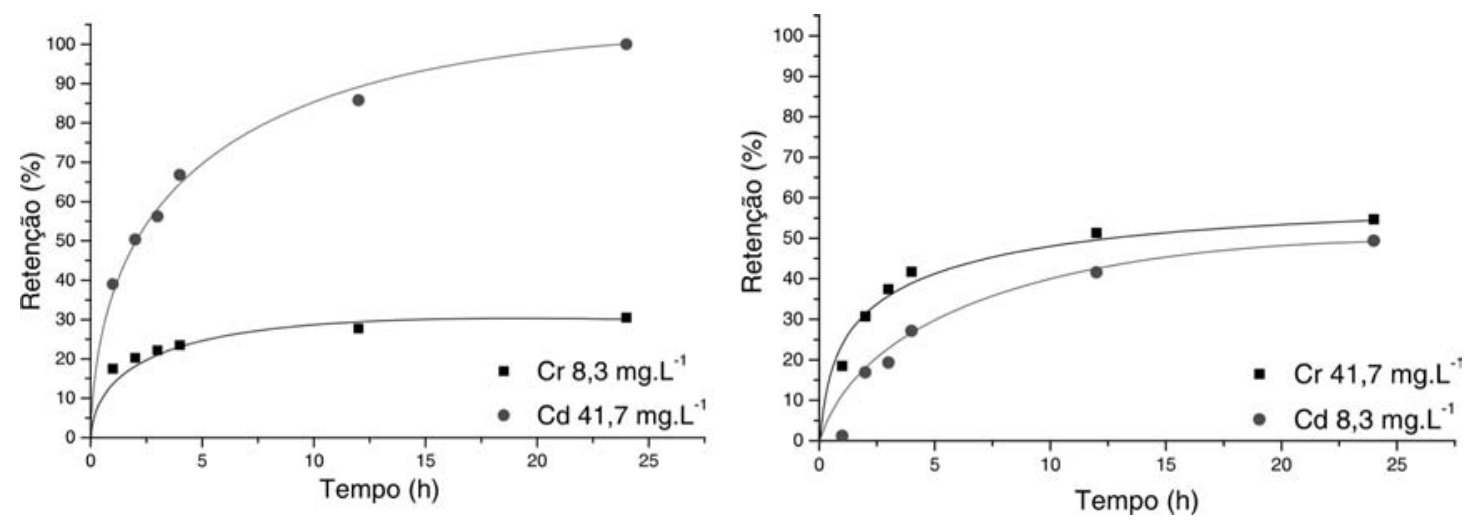

Figura 4 - Cinéticas de adsorção para o par Cr(III)/Cd(II).

Um outro parâmetro a ser definido é o coeficiente de seletividade $K_{B}^{A}$, que inclui a carga dos íons, $\mathrm{z}_{\mathrm{A}}$ e $\mathrm{z}_{\mathrm{B}}$ [17]:

$K_{B}^{A}=\frac{X_{A(z)}^{Z_{B}} \cdot X_{B(s)}^{Z_{A}}}{X_{B(z)}^{Z_{A}} \cdot X_{A(s)}^{Z_{B}}}$

A constante de equilíbrio termodinâmico,

Se os íons possuem a mesma valência $\left(\mathrm{z}_{\mathrm{A}}=\right.$ $\left.\mathrm{z}_{\mathrm{B}}\right)$, então $=\left(\alpha_{B}^{A}\right)^{\mathrm{zA}}$ e para íons univalentes têmse $=\alpha_{B}^{A}$.

O coeficiente de seletividade corrigido, $K_{B}^{\prime A}$ inclui a correção para o coeficiente de atividade dos íons em equilíbrio na solução:

$K_{B}^{\prime A}=\frac{X_{A(z)}^{Z_{B}} \cdot X_{B(s)}^{Z_{A}}}{X_{B(z)}^{Z_{A}} \cdot X_{A(s)}^{Z_{B}}} \cdot \frac{\gamma_{B(s)}^{Z_{A}}}{\gamma_{A(s)}^{Z_{B}}}$

onde $\gamma_{A}$ e $\gamma_{B}$ são os coeficientes de atividade dos íons A e B, respectivamente [7]. Os valores de

$$
\log \gamma=\frac{-0,51 z^{2} \sqrt{\mu}}{1+(a \sqrt{\mu} / 305)}
$$

onde m é a força iônica da solução que contém o íon de carga " $z$ " e de tamanho "a".

$\mathrm{g}_{\mathrm{A}}$ e $\mathrm{g}_{\mathrm{B}}$ podem ser calculados a partir da equação de Debye-Hückel [8]:

$\mathrm{K}_{\mathrm{a}}$, relaciona-se com da seguinte maneira:

$$
K_{a}=K_{B}^{\prime A} \cdot \frac{\gamma_{A(z)}^{Z_{B}}}{\gamma_{B(z)}^{Z_{A}}}
$$

A Tabela 4 apresenta os resultados de coeficiente de seletividade, coeficiente de atividade $\mathrm{g}$, coeficiente de seletividade corrigido, e constante de equilíbrio termodinâmico, $\mathrm{K}_{\mathrm{a}}$, para os pares $\mathrm{Cr}(\mathrm{III}) / \mathrm{Cd}(\mathrm{II})$ e $\mathrm{Ni}(\mathrm{II}) / \mathrm{Mn}(\mathrm{II})$. Na Tabela 5 temos os resultados semelhantes para os pares $\mathrm{Cr}$ (III)/ $\mathrm{Ni}(\mathrm{II})$ e $\mathrm{Cd}(\mathrm{II}) / \mathrm{Mn}(\mathrm{II})$. 
Tabela 4 - Valores de, g, e $\mathrm{K}_{\mathrm{a}}$ para os pares $\mathrm{Cr}(\mathrm{III}) / \mathrm{Cd}(\mathrm{II})$ e Ni(II)/Mn(II).

\begin{tabular}{|c|c|c|c|c|c|c|}
\hline \multicolumn{2}{|c|}{$\begin{array}{c}\text { Concentrações iniciais } \\
\qquad\left(\mathrm{mg} \mathrm{L}^{-1}\right)\end{array}$} & \multirow{2}{*}{$\begin{array}{c}K_{C d}^{C r} \\
1,56\end{array}$} & \multicolumn{2}{|c|}{$\gamma$} & \multirow{2}{*}{$\begin{array}{c}K_{C d}^{\prime C r} \\
2,14\end{array}$} & \multirow{2}{*}{$\begin{array}{c}\mathrm{Ka} \\
1,52\end{array}$} \\
\hline \multirow{6}{*}{$\begin{array}{l}\mathrm{Cr}(\mathrm{III}) / \\
\mathrm{Cd}(\mathrm{II})\end{array}$} & $41,7 / 8,3$ & & 0,624 & 0,810 & & \\
\hline & $33,3 / 16,7$ & 3,85 E-02 & 0,699 & 0,853 & 4,88 E-02 & 3,43 E-02 \\
\hline & $25,0 / 25,0$ & 4,05 E-09 & 0,623 & 0,810 & $5,53 \mathrm{E}-09$ & $4,38 \mathrm{E}-09$ \\
\hline & $16,7 / 33,3$ & $5,76 \mathrm{E}-05$ & 0,685 & 0,845 & 7,40 E-05 & $5,79 \mathrm{E}-05$ \\
\hline & $8,3 / 41,7$ & $8,55 \mathrm{E}-10$ & 0,769 & 0,890 & $1,02 \mathrm{E}-04$ & 7,96 E-04 \\
\hline & & $K_{M n}^{N i}$ & $\mathrm{Ni}(\mathrm{II})$ & $\mathrm{Mn}(\mathrm{II})$ & $K_{M n}^{\prime N i}$ & $\mathrm{Ka}$ \\
\hline \multirow{5}{*}{$\begin{array}{l}\mathrm{Ni}(\mathrm{II}) / \\
\mathrm{Mn}(\mathrm{II})\end{array}$} & $41,7 / 8,3$ & 86,6 & 0,905 & 0,905 & 86,6 & 44,1 \\
\hline & $33,3 / 16,7$ & 5,65 & 0,905 & 0,905 & 5,65 & 3,05 \\
\hline & $25,0 / 25,0$ & 1,36 & 0,913 & 0,913 & 1,37 & 0,78 \\
\hline & $16,7 / 33,3$ & 1,02 & 0,907 & 0,907 & 1,02 & 0,63 \\
\hline & $8,3 / 41,7$ & 0,81 & 0,903 & 0,903 & 0,81 & 0,56 \\
\hline
\end{tabular}

Tabela 5 - Valores de, g, e $\mathrm{K}_{\mathrm{a}}$ para os pares $\mathrm{Cr}(\mathrm{III}) / \mathrm{Ni}(\mathrm{II})$ e $\mathrm{Cd}(\mathrm{II}) / \mathrm{Mn}(\mathrm{II})$.

\begin{tabular}{|c|c|c|c|c|c|c|}
\hline \multicolumn{2}{|c|}{$\begin{array}{c}\text { Concentrações iniciais } \\
\left(\mathrm{mg} . \mathrm{L}^{-1}\right)\end{array}$} & \multirow{2}{*}{$\begin{array}{c}K_{N i}^{C r} \\
2,50 \mathrm{E}+09\end{array}$} & \multicolumn{2}{|c|}{$\gamma$} & \multirow{2}{*}{$\begin{array}{c}K_{N i}^{\prime C r} \\
3,27 \mathrm{E}+09\end{array}$} & \multirow{2}{*}{$\begin{array}{c}\mathrm{Ka} \\
2,12 \mathrm{E}+09\end{array}$} \\
\hline \multirow{6}{*}{$\begin{array}{l}\mathrm{Cr}(\mathrm{III}) / \\
\mathrm{Cd}(\mathrm{II})\end{array}$} & $41,7 / 8,3$ & & 0,664 & 0,833 & & \\
\hline & $33,3 / 16,7$ & $6,14 \mathrm{E}+07$ & 0,631 & 0,813 & $8,31 \mathrm{E}+07$ & $5,58 \mathrm{E}+07$ \\
\hline & $25,0 / 25,0$ & $3,54 \mathrm{E}+06$ & 0,574 & 0,780 & $5,08 \mathrm{E}+06$ & $3,54 \mathrm{E}+06$ \\
\hline & $16,7 / 33,3$ & $4,34 \mathrm{E}+07$ & 0,543 & 0,760 & $6,46 \mathrm{E}+07$ & $4,78 \mathrm{E}+07$ \\
\hline & $8,3 / 41,7$ & $9,02 \mathrm{E}+04$ & 0,514 & 0,741 & $1,39 \mathrm{E}+05$ & $1,06 \mathrm{E}+05$ \\
\hline & & $K_{M n}^{C d}$ & \multicolumn{2}{|c|}{$\mathrm{Cd}(\mathrm{II}) \quad \mathrm{Mn}(\mathrm{II})$} & $K_{M n}^{\prime C d}$ & $\mathrm{Ka}$ \\
\hline \multirow{5}{*}{$\begin{array}{l}\mathrm{Ni}(\mathrm{II}) / \\
\mathrm{Mn}(\mathrm{II})\end{array}$} & $41,7 / 8,3$ & 82,9 & 0,861 & 0,861 & 82,9 & 82,8 \\
\hline & $33,3 / 16,7$ & $8,41 \mathrm{E}+02$ & 0,823 & 0,824 & $8,4 \mathrm{E}+02$ & $8,4 \mathrm{E}+02$ \\
\hline & $25,0 / 25,0$ & $1,1 \mathrm{E}+02$ & 0,795 & 0,795 & $1,1 \mathrm{E}+02$ & $1,1 \mathrm{E}+02$ \\
\hline & $16,7 / 33,3$ & 6,83 & 0,779 & 0,780 & 6,84 & 6,83 \\
\hline & $8,3 / 41,7$ & 54,9 & 0,761 & 0,761 & 55,0 & 55,0 \\
\hline
\end{tabular}


Finalmente, a partir dos valores de $\mathrm{K}_{\mathrm{a}}$ podese determinar a energia livre $\left(\mathrm{DG}^{\circ}\right)[1]$ :

$$
\Delta G^{o}=\frac{-R T}{z_{A} z_{B}} \ln K a
$$

Os resultados obtidos aplicando-se a equação (6) são apresentados na Tabela 6.

Tabela 6 - Valores de energia livre $\left(\Delta \mathrm{G}^{\circ}\right)$.

\begin{tabular}{|c|c|c|}
\hline \multicolumn{2}{|c|}{ Concentrações iniciais $\left(\mathrm{mg} \mathrm{L}^{-1}\right)$} & $\Delta \mathrm{G}^{\mathrm{o}}\left(\mathrm{kJ} \mathrm{mol}^{-1}\right)$ \\
\hline \multirow{5}{*}{$\mathrm{Cr}(\mathrm{III}) / \mathrm{Cd}(\mathrm{II})$} & $41,7 / 8,3$ & $-0,17$ \\
\hline & $33,3 / 16,7$ & 1,39 \\
\hline & $25,0 / 25,0$ & 7,95 \\
\hline & $16,7 / 33,3$ & 4,03 \\
\hline & $8,3 / 41,7$ & 2,95 \\
\hline \multirow{5}{*}{$\mathrm{Ni}(\mathrm{II}) / \mathrm{Mn}(\mathrm{II})$} & $41,7 / 8,3$ & $-2,35$ \\
\hline & $33,3 / 16,7$ & $-0,69$ \\
\hline & $25,0 / 25,0$ & 0,15 \\
\hline & $16,7 / 33,3$ & 0,29 \\
\hline & $8,3 / 41,7$ & 0,36 \\
\hline \multirow{5}{*}{$\mathrm{Cr}(\mathrm{III}) / \mathrm{Ni}(\mathrm{II})$} & $41,7 / 8,3$ & $-8,87$ \\
\hline & $33,3 / 16,7$ & $-7,37$ \\
\hline & $25,0 / 25,0$ & $-6,23$ \\
\hline & $16,7 / 33,3$ & $-7,30$ \\
\hline & $8,3 / 41,7$ & $-4,78$ \\
\hline \multirow{5}{*}{$\mathrm{Cd}(\mathrm{II}) / \mathrm{Mn}(\mathrm{II})$} & $41,7 / 8,3$ & $-2,74$ \\
\hline & $33,3 / 16,7$ & $-4,17$ \\
\hline & $25,0 / 25,0$ & $-2,90$ \\
\hline & $16,7 / 33,3$ & $-1,19$ \\
\hline & $8,3 / 41,7$ & $-2,48$ \\
\hline
\end{tabular}

No par $\mathrm{Cr}(\mathrm{IIII} / \mathrm{Cd}$ (II) ocorre preferência pelo cátion $\mathrm{Cd}(\mathrm{II})$, exceto no sistema contendo elevada concentração de $\mathrm{Cr}$ (III). No par $\mathrm{Ni}(\mathrm{II}) / \mathrm{Mn}$ (II), a preferência por um dos cátions é bem menos pronunciada, havendo uma maior indicação de favorecimento na adsorção do $\mathrm{Ni}(\mathrm{II})$ apenas quando este cátion está presente em elevada concentração. No par $\mathrm{Cr}(\mathrm{III}) /$ $\mathrm{Ni}(\mathrm{II})$ o sistema é espontâneo para a adsorção do
$\mathrm{Cr}$ (III) em todas as concentrações testadas, o mesmo ocorrendo para o Cd(II) no par Cd(II)/Mn(II). Os resultados estão de acordo com as observações feitas anteriormente, na avaliação da cinética de adsorção.

\section{Dessorção} na Figura 5.

Os resultados de dessorção são apresentados

Devido a carga estrutural relativamente elevada da escolecita, foi observada uma reduzida capacidade de troca dos cátions de metais de transição adsorvidos por cátions sódio ou cálcio. O processo de dessorção ocorre predominantemente na primeira hora de contato e, considerando que o modelo de Freundlich é o que mais se adequa ao comportamento observado nas isotermas de adsorção deste material [10], a exemplo do que ocorre com outros materiais nos quais existe mais de um tipo de sítio de troca [2], podemos supor que a troca só ocorre em sítios de fácil acesso, principalmente aqueles localizados na superfície externa da escolecita.

$\mathrm{O}$ Cr(III) foi minimamente dessorvido em ambos os casos. Resultado semelhante foi observado com o uso de philipsita e chabazita [15], sendo a irreversibilidade atribuída a distorções que ocorrem na estrutura da zeólita devido a substituição dos cátions originais, de menor valência, pelo cromo trivalente.

Para os demais cátions, o sódio foi um pouco mais efetivo no processo de dessorção do que o cálcio. Neste caso, tanto o raio iônico hidratado $(3,58$ Å) [13] quanto a energia de hidratação ( $\left.406 \mathrm{~kJ} \mathrm{~mol}^{-1}\right)$ [19] do sódio favorecem a troca quando comparado com o cálcio, onde temos os valores de 4,12 ̊e 1577 $\mathrm{kJ} \mathrm{mol}^{-1}$, respectivamente. No caso do Cd(II), observamos elevados valores de dessorção frente aos cátions sódio. Este fato já havia sido observado para outras zeólitas naturais, como chabazita, philipsita, mordenita e ferrierita e parece ser independente da carga estrutural da zeólita ou do raio iônico hidratado e da energia de hidratação do cátion [5]. Tanto a adsorção preferencial do $\mathrm{Cd}$ (II) em relação ao $\mathrm{Cr}$ (III) quanto o seu comportamento frente a dessorção podem estar relacionados ao fato de que íons de metais de transição com distribuição d [10] em sua camada de valência e baixos estados de oxidação, como o $\mathrm{Cd}(\mathrm{II})$, são altamente lábeis e, portanto, atingem rapidamente situações de equilíbrio [16]. 

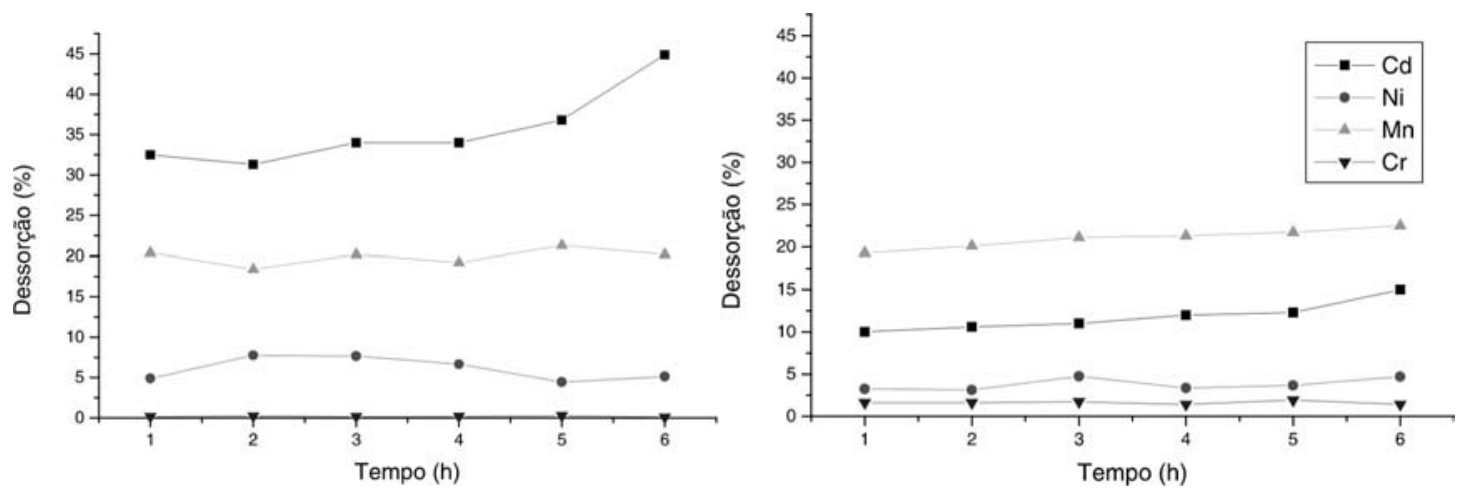

Figura 5 - Cinéticas de dessorção com $\mathrm{NaCl}$ (esquerda) e $\mathrm{Ca}\left(\mathrm{NO}_{3}\right)_{2}$ (direita).

\section{Conclusões}

A escolecita apresentou uma elevada eficiência de adsorção dos cátions de metais pesados $\mathrm{Mn}(\mathrm{II}), \mathrm{Cd}(\mathrm{II}) \mathrm{Ni}(\mathrm{II})$ e $\mathrm{Cr}(\mathrm{III})$. Vários fatores influenciaram a competitividade de adsorção entre dois cátions presentes em solução, destacando-se que a valência elevada e o raio iônico hidratado reduzido favorecem a adsorção, o que está de acordo com o comportamento de trocadores iônicos com elevada carga estrutural.

Nos testes de dessorção, a escolecita demonstrou uma elevada capacidade de reter os cátions $\mathrm{Ni}$ (II) e $\mathrm{Cr}$ (III) em sua estrutura, enquanto o Cd(II) apresentou comportamento diferenciado, sendo adsorvido e dessorvido da estrutura zeolítica com facilidade.
Aliado aos fatos desta zeólita ocorrer em abundância e de não ser impactante ao meio ambiente, a sua seletividade e elevada capacidade de adsorção torna o seu uso na retenção de metais pesados de efluentes aquosos potencialmente útil econômica e ecologicamente.

\section{Agradecimentos}

Os autores agradecem ao PIBIC/CNPq pelas bolsas concedidas.

Recebido em: 25/08/03

Aceito em: 23/10/03

S. M. Dal Bosco, R. S. Jimenez, W. A. Carvalho. Removal of heavy metals from industrial effluents by scolecite: competition and desorption processes.

\begin{abstract}
During recent years stringent regulations of wastewater discharge into aquatic bodies have been imposed. Removal of contaminants, as heavy metals, is one of the fundamental goal in waste treatment. In order to achieve efficient cost effective technology, natural materials as zeolites are generally been applied is wastewater treatment to remove pollutants. We investigated the ability of scolecite, a natural zeolite from the top of the basaltic flows of Serra Geral Formation in Paraná Basin, Brazil, to retain chromium(III), nickel(II), cadmium(II) and manganese(II) in synthetic aqueous effluents. We evaluated the ion selectivity by batch experiments in a binary combination solution. The scolecite showed high efficiency retaining the tested metals, even when they are in competition. The zeolite preference for one cation could be related to cations with high charge density (e.g., $\mathrm{Cr}(\mathrm{III})$ ) and lower hydration energies (e.g., Cd(II)). The unusual Cd(II) exchange behavior observed can be related to its lability. $\mathrm{Cr}(\mathrm{III})$ and $\mathrm{Ni}(\mathrm{II})$ were efficiently adsorbed, so they could not be substantially removed from scolecite structure by sodium or calcium cations.
\end{abstract}

Keywords: cation exchange; heavy metals; scolecite. 


\section{Referências}

[1] A. W. Adamson, A. P. Gast, Physical Chemistry of Surfaces, Wiley, New York, 6th edn., 1997, p. 641.

[2] L. Barrer, Cation Exchange Equilibria in Zeolites and Feldspatoids. In: L. B. Sand, F. A. Mumpton, Eds, Natural Zeolites, Occurrence, Properties, Use. Pergamon Press, Oxford, 1978, p. 385.

[3] D. W. Breck, Zeolite Molecular Sieves, Wiley, New York, 1974.

[4] W. A. Carvalho, R. L. Mincato, L. A. S. Silva, Rev. Tecnol., v. esp. (2000) 25.

[5] C. Colella, Miner. Depos. 31 (1996) 554.

[6] M. T. Costa Rupp, E. F. Souza-Aguiar, Troca Iônica. In: Curso Iberoamericano sobre Peneiras Moleculares, 2, 1995, São Carlos, p.397.

[7]Z. Hagiwara, M. Uchida, Ion-Exchange Reactions of Processed Zeolite and its Application to the Removal of Ammonia Nitrogen in Wastes, Natural Zeolites, Pergamon Press, Oxford, 1976.

[8] D. C. Harris, Quantitative Chemical Analysis, W. H. Freeman, New York, 5th edn., 1999.

[9] D. A. Holmes, Zeolites, In: D. D. Carr, Ed., Industrial Min- eral and Rocks. Society for Mining Metallurgy and Exploration, Littleton, 1994, p. 1129.

[10] R. S. Jimenez, S. M. Dal Bosco, W. A. Carvalho, Mater. Res. 2003, Submetido.

[11] K. J. Murata, M. L. L. Formoso, A. Roisenberg, J. Geology. 95 (1987) 455.

[12] C. C. Nerbitt, T. E. Davis, Removal of Heavy Metals from Metallurgical Effluents by the Simultaneous Precipitation and Flotation of Metal Sulfides Using Column Cells. In: Extraction and processing for the treatment and minimization of waste, The Mineral, Metals and Materials Society, Pennsylvania, 1994, p. 331.

[13] E. R. Nightingale Jr, J. Phys. Chem. 63 (1959) 1381.

[14] S. K. Ouki, M. Kavannagh, Wat. Sci. Tech. 39 (1999) 115.

[15] M. Pansini, C. Colella, M. de Gennaro, Desalination, 83 (1991) 145.

[16] D. F. Shriver, P. W. Atkins, Química Inorgânica, Bookman, Porto Alegre, 3 ed., 2003.

[17] Y. Tanaka, M. Tsuji, Materials Res. Bull. 32 (1997) 461.

[18] A. Vogel, Análise Química Quantitativa, 5 ed., LTC, Rio de Janeiro, 1992, $712 \mathrm{p}$.

[19] G. Wulfsberg, Principles of Descriptive Chemistry, Brooks/ Cole Pub., Monterey CA, 1987, p. 23. 